\title{
CRY2 Gene
}

National Cancer Institute

\section{Source}

National Cancer Institute. CRY2 Gene. NCI Thesaurus. Code C106139.

This gene is involved in circadian rhythm. 